\title{
AURICULOTERAPIA NO TRATAMENTO DA OBESIDADE NA ATENÇÃO BÁSICA
}

\section{AURICULOTHERAPY IN THE TREATMENT OF OBESITY IN BASIC CARE}

Sandra Patrícia de Oliveira Vilaça ${ }^{1}$

Diógenes José Gusmão Coutinho²

\begin{abstract}
Resumo: Esse estudo objetivou ampliar o cuidado das pessoas com obesidade que participaram do atendimento coletivo da equipe de matriciamento do Núcleo Ampliado da Saúde da Família na Atenção Básica (NASF-AB), com a inserção das Práticas Integrativas e Complementares no SUS (PICs), utilizando a prática da auriculoterapia como possibilidade terapêutica na Estratégia de Saúde da Família (ESF), essa prática foi aplicada a trinta usuários do serviço de uma Unidade básica de Saúde com diagnóstico de obesidade, que participaram de cinco encontros consecutivos, com abordagem quantitativa e qualitativa, usando pontos reflexos para redução de peso e ansiedade, e como resultado foi a redução do grau de obesidade e intensidade do apetite nos participantes.
\end{abstract}

Palavras-Chave: Atenção Básica. Auriculoterapia. Obesidade- Práticas Integrativas e Complementares.

ABSTRACT: This study aimed to expand the care of people with obesity who participated in the collective care of the Matrix Team of Extended Family Health in Primary Care (NASF-AB), with the insertion of Integrative and Complementary Practices in SUS (PICs), using the practice of auriculotherapy as a therapeutic possibility in the Family Health Strategy (FHS), this practice was applied to thirty users of the service of a basic health unit diagnosed with obesity, who participated in five consecutive meetings, with quantitative and qualitative approach, using reflex points for weight reduction and anxiety, and as a result was a reduction in the degree of obesity and appetite intensity in the participants.

Keywords: Primary Care. Auriculotherapy. Obesity. Integrative and Complementary Practices.

\section{INTRODUÇÃO}

\footnotetext{
${ }^{1}$ Graduada em Nutrição, Aluno de Especialização em Pesquisa Avançada pela Faculdade Alpha, Mestrado Internacional pela Atenas College University. E-mail: sp.vil.aca@hotmail.com.

2 Graduado em Biologia pela UFRPE. Doutor em Biologia pela UFPE. E-mail: alphadiogenes@gmail.com.
} 


\section{Universidade do Extremo Sul Catarinense \\ Revista lbero-Americano de Humanidades, Ciências e \\ Educação \\ Unesc Produção e democratização do conhecimento na lbero-América}

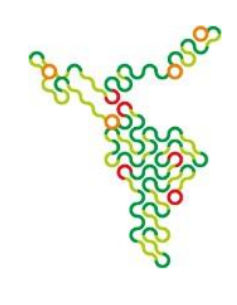

A auriculoterapia é um sistema médico integral, originado a milhares de anos na china. Utiliza a linguagem que retrata simbolicamente as leis da natureza e que valoriza a inter-relação harmônica entre as partes visando à integralidade. Como fundamento aponta a teoria ying-yang e dos cinco elementos (madeira, fogo, terra, metal e água), esse sistema fazem parte de um conjunto de práticas e saberes agrupados pela característica comum de não participarem do escopo da medicina convencional. (BRASIL, 2006).

As Práticas Integrativas e Complementares (PIC) é a designação que o Ministério da Saúde (MS) deu ao que se tem chamado na literatura Científica Internacional de Medicinas Alternativas e Complementares. Referese a um conjunto heterogêneo de práticas, produtos e saberes, agrupados pela característica comum de não pertencerem ao escopo dos saberes/práticas consagrados na medicina convencional. (SOUSA, 2017). O desenvolvimento das PIC nos Sistemas de Saúde públicos universais é favorável e seu crescimento e incontestável nas últimas décadas. Desde a Conferência Internacional de Alma Ata, realizada em 1978, a Organização Mundial da Saúde (OMS) recomenda a seus países membros a inclusão das PIC nos Sistemas Públicos de Saúde. (JÚNIOR, 2016).

Assim, as terapias alternativas e complementares vêm sendo gradativamente inseridas no Sistema Único de Saúde (SUS), como preveem as Políticas Nacionais de Promoção da Saúde e de Práticas Integrativas e Complementares (PNPIC) no SUS (Portarias Ministeriais no 687 de 30 de março de 2006, o 971 em 03 de maio de 2006, no 1.600, de 17 de julho de 2006 e no 154, de 24 de janeiro de 2008). A PNPIC legitimou expressamente as práticas da fitoterapia, da homeopatia, da medicina tradicional chinesa, medicina antroposófica e do termalismo social, mas também significou um impulso no reconhecimento e crescimento de todas as demais PIC no SUS. (BRASIL, 2012).

A inclusão das PIC no SUS é apropriada aos diversos níveis de atenção 


\section{Universidade do Extremo Sul Catarinense \\ Revista Ibero-Americano de Humanidades, Ciências e \\ Educação \\ Unesc Produção e democratização do conhecimento na lbero-América}

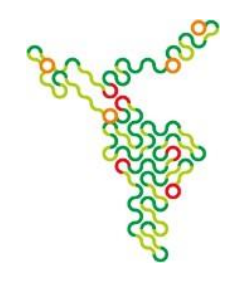

à saúde, com ênfase na inserção na Atenção Básica, na perspectiva da prevenção de agravos e da promoção e recuperação da saúde, propiciando um cuidado continuado, humanizado e integral. (BRASIL, 2008). Há mais de 30 anos a prevalência de sobrepeso e obesidade vem crescendo, e nesse cenário epidemiológico a obesidade é um fator de risco para outras doenças como hipertensão, diabetes, câncer, entre outras. A OMS define a obesidade como condição crônica caracterizada pelo acúmulo excessivo de gordura que traz repercussões à saúde. Portanto, é categorizada, na 10a revisão da Classificação Internacional de Doenças (CID-10), no item de doenças endócrinas, nutricionais e metabólicas. (DIAS, ANJOS e BURLANDY, 2017).

A política nacional de Alimentação e Nutrição tem em seus propósitos a promoção de práticas alimentares e modos de vida saudáveis e nesse contexto a prevenção e o tratamento da obesidade configura-se grande desafio. (BRASIL, 2003). Considerando o pluralismo em cuidados de saúde integrando abordagens que ampliam as possibilidades diagnóstico-terapêuticas, o uso da auriculoterapia no tratamento da obesidade vem ganhando espaço, são praticadas por profissionais de saúde da família, assim como os profissionais da equipe de matriciamento do Núcleo Ampliado de Saúde da Família e Atenção Básica (NASF-AB), com formação prévia dessa prática, contribuindo para a ampliação da clínica, é uma prática de baixo custo, facilita a participação do usuário do serviço, e uma maior consideração da subjetividade do sujeito, com impacto favorável na Atenção Básica. Ela pode ser usada tanto nos atendimentos individuais como nos atendimentos coletivos, como possível resposta aos limites e lacunas da biomedicina, e pode ser considerada estratégia para o desenvolvimento da universalidade, equidade e integralidade. (SOUSA e VIEIRA, 2005).

A auriculoterapia é um tipo de tratamento terapêutico que consiste em estimular os pontos cutâneos da orelha, objetivando a homeostase interna do organismo, estimulando o processo de autocura das pessoas. (LEVIN e 


\section{Universidade do Extremo Sul Catarinense \\ Revista Ibero-Americano de Humanidades, Ciências e \\ Educação \\ Unesc Produção e democratização do conhecimento na lbero-América}

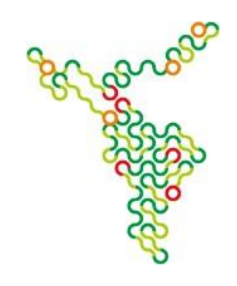

JONAS, 2011). Hoje, o conhecimento do ser humano, tem sido o ponto de estudo para que seja revista a posição da medicina como a única regeneradora e mantedora da saúde humana, é culturalmente aceita, de grande procura, com favorecimento de vínculo terapeuta-usuário, contribuindo para um olhar mais ampliado do sujeito, incluindo dimensões psicossociais, espirituais e subjetivas, entre muitas de suas vantagens é um método não invasivo, com baixo risco de infecção, maior área de abrangência dos pontos, manipulação simples, o paciente pode retirar a aplicação em casa e os efeitos da aplicação podem durar até sete dias.

Esse estudo objetivou ampliar o cuidado das pessoas com obesidade que participaram do atendimento coletivo da equipe de matriciamento do Núcleo Ampliado da Saúde da Família na Atenção Básica (NASF-AB), com a inserção das Práticas Integrativas e Complementares no SUS (PICs), utilizando a prática da auriculoterapia como possibilidade terapêutica na Estratégia de Saúde da Família (ESF), nesses encontros, observou-se nas falas dos usuários, que um dos grandes motivos para não aderirem a reeducação alimentar era o comportamento de muito apetite, onde muitas vezes haviam sofrimento, ansiedade, tentativas de dietas fracassadas, falta de motivação e estímulo, autocuidado insuficiente, motivos pelos quais podem ser aliviados com o uso da auriculoterapia. A partir deste problema de pesquisa, foi sugerido que os mesmos aderissem ao tratamento, com finalidade de aumento da saciedade e redução da fome. $O$ trabalho em questão se justifica pela magnitude e relevância que a obesidade assume no cenário brasileiro, e as abordagens com os usuários são pouco resolutivas e ineficazes. Existindo poucos trabalhos sobre esse tema.

\section{METODOLOGIA}

Trata-se de um estudo tipo descritivo com abordagem qualitativa e quantitativa, realizada com pessoas, devidamente cadastradas na unidade de saúde da família do Bairro Novo 1, situado no município de Camaragibe-PE, 


\section{Universidade do Extremo Sul Catarinense \\ Revista Ibero-Americano de Humanidades, Ciências e \\ Educação \\ Unesc Produção e democratização do conhecimento na lbero-América}

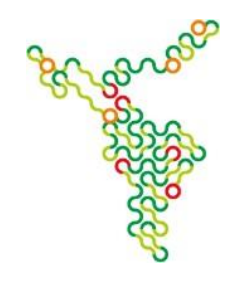

onde foi o campo de pesquisa, que foram consultadas pela enfermeira da família e encaminhadas para o atendimento coletivo da nutricionista do NASF$A B$, com diagnóstico de obesidade.

A amostra foi composta por trinta usuários, adultos, que não esteja usando remédios para emagrecer, nem para ansiedade e depressão, com diagnóstico de obesidade, que necessitavam do cuidado nutricional específico, triado pela enfermeira da USF (Unidade de Saúde da Família), no mês de janeiro 2019, os mesmos foram encaminhados para o primeiro encontro na sala de reuniões da unidade, onde foi realizado uma roda de conversas sobre suas potencialidades e possibilidades e reconhecimento da sua situação de saúde pela nutricionista, com abordagem sobre as PICS, e sobre o uso da auriculoterapia como tratamento da obesidade, redução da circunferência da cintura e o IMC (Índice de Massa Corporal), e o apetite. Para participar os usuários assinaram consentimento livre e esclarecido conforme com a Resolução 466/12 Cap. IV inciso IV. 1, estavam na faixa etária de adultos de ambos os sexos, com diagnóstico de obesidade, triado pela enfermeira.

Os dados foram coletados no primeiro e último contato, utilizando como instrumento a ficha de anamnese em auriculoterapia onde apresenta as mesmas questões para todos os usuários, garantindo 0 anonimato, apresentando questões abertas e fechadas (Apêndice A). Os dados quantitativos serão antropométricos (peso, altura, circunferência da cintura, Índice de massa corporal o IMC), perfil da demanda atendida (sexo, faixa etária, doenças diagnosticadas previamente) contendo medidas iniciais e finais ao tratamento e a avaliação do apetite (fome) onde a numeração um representava muito pouco, dois pouco, três regular, quatro muito e cinco bastante fome. Para técnica qualitativa será utilizado análise documental (prontuário), e observação participante.

Os dados quantitativos coletados serão organizados e analisados de acordo com a frequência simples e proporcionalidade das variáveis 


\section{Universidade do Extremo Sul Catarinense \\ Revista lbero-Americano de Humanidades, Ciências e \\ Educação \\ Unesc Produção e democratização do conhecimento na lbero-América}

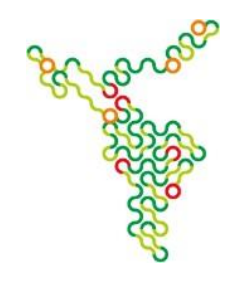

construídas. Para a análise qualitativa, haverá a sistematização e validação dos dados. O plano de análise consiste na correlação dos objetivos, as condições verificadoras, métodos de coletas e análise de dados. Aspectos Éticos: Resolução 466/12. CNS/MS.

Foram realizados cinco encontros, no primeiro encontro foi aplicada a ficha de anamnese em auriculoterapia e coletado os dados, pactuado o acordo de convivência, dinâmica de apresentação, realizado o acolhimento e roda de conversa com mediação da nutricionista, com duração de 1 hora, em seguida encaminhado para sessão de auriculoterapia, os três encontros seguintes com intervalo de uma semana, foram apenas de sessão de auriculoterapia, e o último foram coletados os mesmos dados para comparação, e avaliação participante.

O material utilizado na técnica foi a placa de auriculoterapia (plástica para serem preparadas com as sementes facilitando na aplicação dos pontos auriculares), estilete (usado na preparação das placas de auriculoterapia), álcool 70으, algodão, Sementes de mostarda (comestível), fita microporosa (preferência bege), pinça anatômica pequena serrilhada, apalpador de auriculoterapia A técnica de auriculoterapia usada consiste na fixação de sementes de mostarda com esparadrapo tipo micropore de cor bege, sendo apalpado o ponto reflexo com instrumentos próprio (apalpador), este movimento de pressão auxilia a identificar pontos mais sensíveis e doloridos à palpação indicando possível enfermidade na área correspondente, o paciente na posição sentado, com o máximo de relaxamento.(HONG, 2005).

Cada participante ficou com as sementes na orelha por cerca de sete dias e orientado a fazer a própria estimulação três vezes ao dia por dez segundos em cada ponto. Sendo alternada a aurícula, ou seja, uma semana a aurícula direita e na semana seguinte à esquerda, e assim consecutivamente até completar o fim do tratamento, os encontros foram realizados semanalmente. Para realização da técnica, foi observado o local se estava com 


\section{Universidade do Extremo Sul Catarinense \\ Revista lbero-Americano de Humanidades, Ciências e \\ Educação \\ Unesc Produção e democratização do conhecimento na lbero-América}

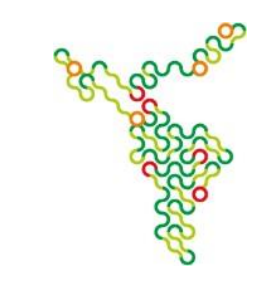

boa iluminação, e limpeza do pavilhão auricular com álcool 70\%. Portanto, os pontos ou áreas reflexas auxiliam na avaliação diagnóstica e são utilizados no tratamento das enfermidades, na figura 1, observamos os pontos ou áreas reflexas existentes no pavilhão auricular. (HANS-ULRICH, 2007).

Figura 1- pontos ou áreas reflexas existentes no pavilhão auricular

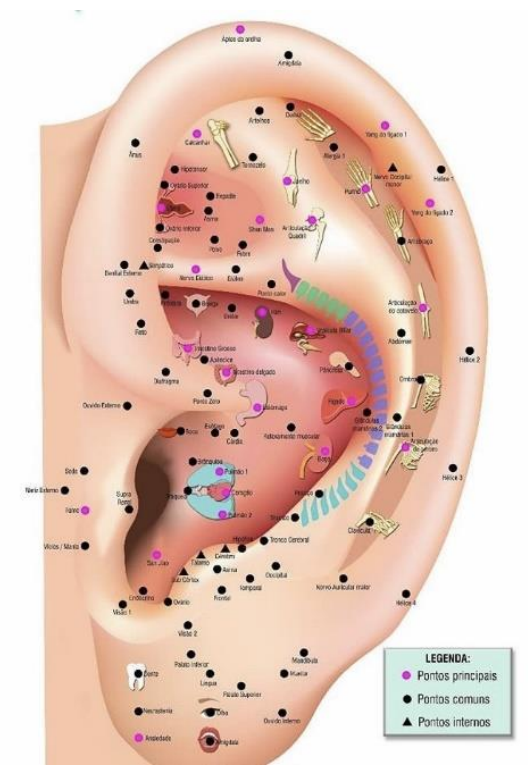

Fonte: UFSC, 2016.

Para este estudo, vamos utilizar os pontos reflexos relacionados com o problema de pesquisa, como citado na fala dos usuários, onde o fracasso na perda de peso eram a ansiedade, aumento da fome, dietas fracassadas, falta de estímulo, autocuidado insuficiente.

No canto interno e inferior do lóbulo da orelha (primeiro ponto do estudo), encontramos o ponto da ansiedade que estimulado podemos tratar a ansiedade, agitação, insônia, estresse emocional e irritabilidade. Na cavidade da concha da orelha (Cava) encontramos o ponto San Jiao referente o metabolismo (segundo ponto do estudo), com ação de tratamento para constipação, indigestão, anemia, obesidade, enxaqueca, vertigens e dores intercostais. Ainda na Cava encontramos o ponto do coração (terceiro ponto do estudo), com ação de tratamento para palpitação, taquicardia, hipertensão, 


\section{Universidade do Extremo Sul Catarinense \\ Revista lbero-Americano de Humanidades, Ciências e \\ Educação \\ Unesc Produção e democratização do conhecimento na lbero-América}

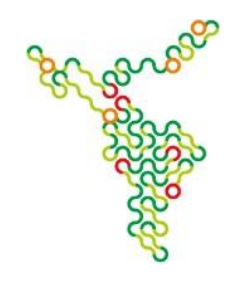

ansiedade, depressão, insônia e dispneia.

$\mathrm{Na}$ cimba da concha, encontramos o ponto do fígado (quarto ponto do estudo), com ação de tratamento como transtornos gastrointestinais, hematológico, cutâneos e oculares como: vômito, constipação, gastrite, distensão abdominal, doenças hemorrágicas, dermatites, eczemas, depressão, irritabilidade e estresse. No ramo da Hélice, encontramos o ponto do estômago (quinto ponto do estudo) com ação de tratamento Problemas gástricos, estomatite, gastralgia, vômitos, hiperemese gravídica, inapetência, náuseas. Na estrutura anatômica do trago, encontramos o ponto da fome e sede (sexto e sétimo ponto do estudo) com ação de tratamento de Compulsão alimentar, obesidade, inapetência e anorexia nervosa, polidipsia, desidratação, sede relacionado a hiperglicemia, poliúria e enurese. Na incisura intertágica, encontramos o ponto endócrino (oitavo ponto do estudo) com ação de tratamento de todos os transtornos endócrinos, hipo e hipertiroidismo, diabetes, transtornos ginecológicos e reumatoides. E por último o Shen Men na fossa triangular (nono ponto do estudo) com ação de tratamento de ansiedade, distúrbios mentais, estabilização emocional, em condições de dor e possui atividade anti-inflamatória. (NEVES, 2014); (ROMOLI, 2013).

\section{FUNDAMENTAÇÃO TEÓRICA}

A Organização Mundial da Saúde (OMS) define obesidade como uma doença crônico-degenerativa caracterizada pelo excesso de gordura corporal resultante de um desequilíbrio energético. (OMS. 2016). De acordo com o Ministério da Saúde (MS), o balanço energético positivo é o determinante mais imediato do acúmulo excessivo de gordura e ocorre quando a quantidade de energia consumida é maior que a quantidade de energia gasta. Entretanto existem várias maneiras de classificar ou diagnosticar a obesidade, dentre elas a mais utilizada é o Índice de Massa Corporal (IMC ou Índice de Quetelet), utilizando-se a seguinte fórmula: IMC = Peso atual $(\mathrm{kg}) /$ altura $(\mathrm{m} 2)$. (BONIZOL, et al., 2016).

A classificação adaptada pela Organização Mundial da Saúde (OMS), 


\section{Universidade do Extremo Sul Catarinense}

Revista Ibero-Americano de Humanidades, Ciências e

Educação

UnesC Produção e democratização do conhecimento na lbero-América

apresentada na tabela 1, baseia-se em padrões internacionais desenvolvidos para pessoas adultas descendentes de europeus.

Tabela 1- classificação de peso pelo IMC

\begin{tabular}{|l|l|}
\hline Classificação & $\mathrm{IMC}\left(\mathrm{kg} / \mathrm{m}^{2}\right)$ \\
\hline Baixo peso IMC $\left(\mathrm{kg} / \mathrm{m}^{2}\right)$ & $<18,5$ \\
\hline Peso normal & $18,5-24,9$ \\
\hline Sobrepeso & 25,0 a 29,9 \\
\hline Obeso I & 30,0 a 34,9 \\
\hline Obeso II & 35,0 a 39,9 \\
\hline Obeso III & $\geq 40$ \\
\hline
\end{tabular}

Fonte: Organização Mundial da Saúde (OMS),

Mais recentemente, tem-se notado que a distribuição de gordura é mais preditiva de saúde. A combinação de massa corporal e distribuição de gordura é, provavelmente, a melhor opção para preencher a necessidade de avaliação clínica. Para o estudo de distribuição corporal pode-se usar a medição da espessura das pregas cutâneas, biopedância, ultrassonografia, tomografia computadorizada, ressonância magnética, relação circunferência abdominalquadril e medida da circunferência abdominal. A OMS estabelece como ponto de corte para risco cardiovascular aumentado: medida de circunferência abdominal igual ou superior a $94 \mathrm{~cm}$ em homens e $80 \mathrm{~cm}$ em mulheres caucasianos. (ABESO, 2009)

O tratamento da obesidade é complexo e multidisciplinar, há várias opções de tratamento como o farmacológico, o dietético, a terapia cognitivocomportamental, cirúrgico, etc., porém a aderência e resultados dos tratamentos convencionais não são satisfatórios, e como recurso complementar de tratamento podemos citar a medicina Alternativa e complementar, estas práticas podem ser chamadas de "complementares", quando utilizadas em 


\section{Universidade do Extremo Sul Catarinense \\ Revista lbero-Americano de Humanidades, Ciências e \\ Educação \\ Unesc Produção e democratização do conhecimento na lbero-América}

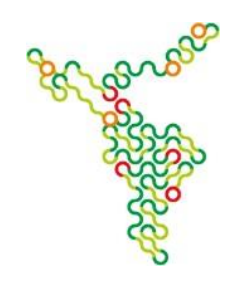

associação à biomedicina; "alternativas", quando empregadas em substituição à prática biomédica; e, por fim, "integrativas", quando são usadas conjuntamente à biomedicina, considerando que há evidências científicas de segurança e efetividade.

Uma dessas práticas e a Medicina Tradicional Chinesa (MTC), onde o equilíbrio energético significa a harmonia do homem com uma infinita rede de fluxos de energias entrelaçados no universo, ou seja, o homem transita na rede de energia existente no universo seguindo seu Tao (caminho) e assim, encontra o equilíbrio. Porém, quando ocorre o desvio do seu Tao, surge o desequilíbrio energético, caracterizando doenças. Para a MTC a obesidade traduz um desequilíbrio energético, o qual é consequência de fatores como: tipo e a quantidade de alimentos consumidos, regularidade da alimentação e estado emocional enquanto se alimenta. (ORNELA et al., 2016).

Na Medicina Tradicional Chinesa a acupuntura é uma terapêutica, que vem sendo amplamente aplicado no Ocidente, em razão de sua fácil aplicação, baixo custo e descrença em tratamentos convencionais. A auriculoterapia é uma terapia pela aurícula, é uma técnica do ramo da acupuntura, que é reflexiva se baseia na correlação das regiões do pavilhão auricular com os órgãos e regiões do corpo. Pontos ou zonas (áreas) auriculares reflexas são locais específicos localizados na superfície do pavilhão auricular que refletem e estão conectados a atividade funcional do organismo como um todo. No caso de um tratamento para apenas uma queixa, poderá ser escolhido um ou dois pontos reflexos, mas se o paciente apresentar diversas queixas, pode-se selecionar em média de 8 até 10 pontos auriculares, sempre priorizando a queixa principal. No caso do tratamento da Obesidade, usamos um protocolo com nove pontos: ansiedade, metabolismo, coração, fígado, estomago, fome, sede, endócrino, Shen Men. Na figura 2 encontra-se a utilização da técnica de auriculoterapia na Atenção Básica na seleção de pontos. 


\section{Universidade do Extremo Sul Catarinense}

Revista Ibero-Americano de Humanidades, Ciências e

Educação

UnesC Produção e democratização do conhecimento na lbero-América

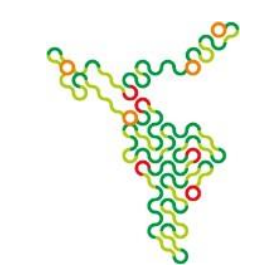

Figura 2- seleção de pontos de auriculoterapia na Atenção Básica

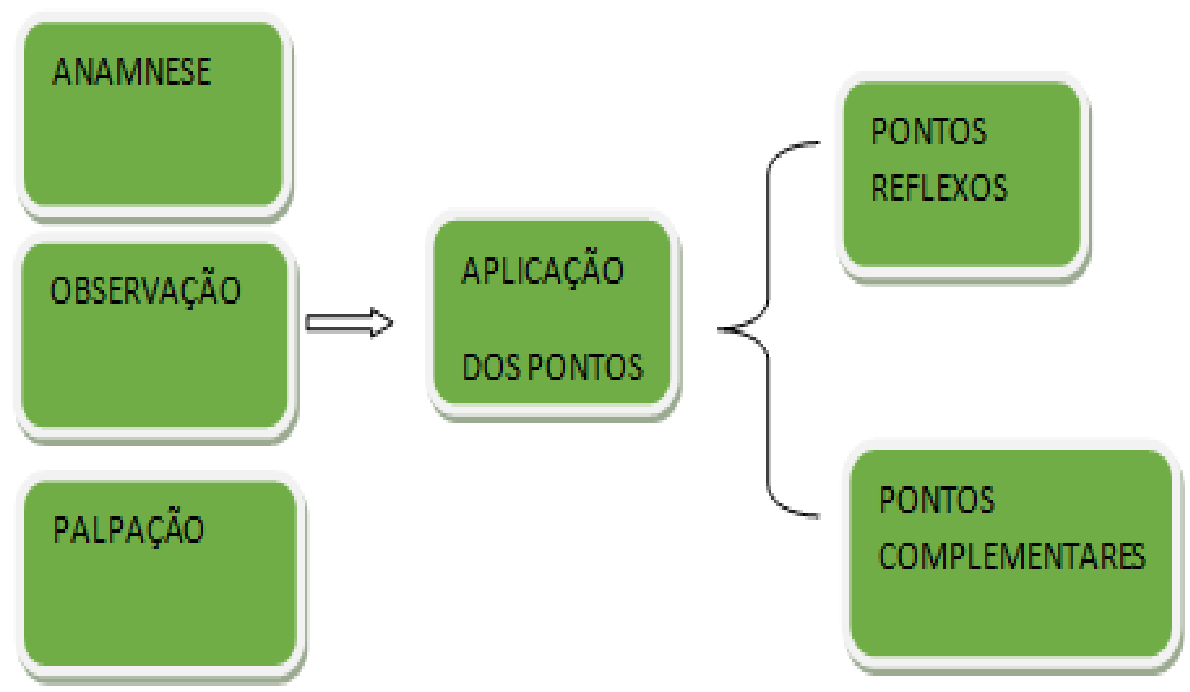

Fonte: UFSC, 2016

\section{RESULTADOS E DISCUSSÃO}

$\mathrm{Na}$ tabela 2, apresenta o perfil dos participantes no primeiro encontro, observa-se uma prevalência de $100 \%$ de indivíduos do sexo feminino, alguns autores relatam o motivo ser a marca cultural do homem à prevenção e o seu autocuidado, fazendo com que este chegue ao serviço apenas em caso de urgência, já que ao longo da trajetória seu cuidado geralmente é mediado pela figura feminina como mãe, esposa e filhas. (GOMES, et al., 2011). Outros fatores seriam insuficiência de ações específicas voltadas para homens na Atenção Básica, a inserção em mercado formal versus horários de funcionamento das Unidades de Saúde, falta de tempo para agendar consultas. (FERRAZ e KRAICZYK, 2010); (BRASIL, 2009).

A faixa etária do grupo estudado variou de 30 anos a idade mínima e 58 anos a idade máxima, com renda de até um salário mínimo, e com diagnóstico de obesidade, que já tinham previamente triado pela enfermeira da unidade de saúde. Para intensidade do apetite (IA), obtido pela escala de percepção autodeclarada contida na ficha de anamnese (Apêndice A), no primeiro 


\section{Universidade do Extremo Sul Catarinense}

Revista Ibero-Americano de Humanidades, Ciências e

Educação

Unesc Produção e democratização do conhecimento na lbero-América

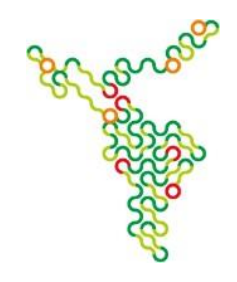

encontro o apetite variou de muito a bastante.

Tabela 2- perfil dos participantes no primeiro encontro do tratamento

\begin{tabular}{lll}
\hline Faixa etária & mínimo 30 anos & máximo 58 anos \\
Renda familiar & $100 \%$ até um salário & $0 \%$ maior que um salário \\
Sexo & $100 \%$ feminino & $0 \%$ masculino \\
Grau de obesidade & $60 \%$ grau I & $40 \%$ igual ou maior que grau II \\
Circunferência abdominal & $100 \%$ maior que $80 \mathrm{~cm}$ & $0 \%$ inferior de $80 \mathrm{~cm}$ \\
Intensidade do apetite & $60 \%$ bastante & $40 \%$ muito \\
\hline
\end{tabular}

Fonte: dados da pesquisa

Ao término do tratamento observou-se, redução no grau de obesidade, Circunferência Abdominal (CA) e intensidade do apetite, conforme a tabela 3.

Tabela 3. Distribuição dos participantes segundo grau de obesidade, circunferência abdominal

(CA) e Intensidade do Apetite (IA), no início e término do tratamento

\begin{tabular}{|c|c|c|c|c|}
\hline & \multicolumn{2}{|c|}{ Início do tratamento } & \multicolumn{2}{|c|}{ Término do tratamento } \\
\hline & $\mathbf{N}$ & 96 & $\mathbf{N}$ & $\%$ \\
\hline \multicolumn{5}{|l|}{ Grau de obesidade } \\
\hline Peso normal & 0 & 0 & 0 & 0 \\
\hline Sobrepeso & 0 & 0 & 5 & 16,67 \\
\hline Grau 1 & 18 & 60.00 & 16 & 53,33 \\
\hline Grau II & 08 & 26.67 & 06 & 20,00 \\
\hline Grau III & 04 & 13,33 & 03 & 10,00 \\
\hline \multicolumn{5}{|l|}{ CA } \\
\hline Maior que $80 \mathrm{~cm}$ & 30 & 100 & 30 & 100 \\
\hline \multicolumn{5}{|l|}{ IA } \\
\hline Bastante & 18 & 60 & 0 & 0 \\
\hline Muito & 12 & 40 & 0 & 0 \\
\hline Regular & 0 & 0 & 20 & 66,67 \\
\hline Pouco & 0 & 0 & 10 & 33,33 \\
\hline Muito pouco & 0 & 0 & 0 & 0 \\
\hline
\end{tabular}

Fonte: dados da pesquisa

Ao término do tratamento podemos observar uma redução no 


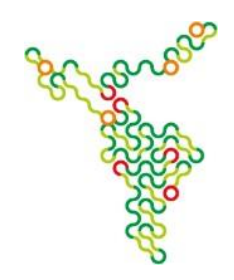

grau de obesidade dos participantes do grupo, embora ao final da pesquisa ainda prevaleceu o sobrepeso e obesidade, sendo esse achado devido ao fato que a duração do tratamento foram cinco semanas, tempo curto para ter uma perda de peso bastante significativa. O mesmo acontece com a circunferência abdominal, onde $100 \%$ dos participantes continuavam acima dos $80 \mathrm{~cm}$, apresentando risco cardiovascular. Em relação a intensidade do apetite, houve uma redução de muito a bastante para pouco ou, muito pouco conforme tabela 3.

A Organização Mundial da Saúde (OMS) estima que pelo menos um bilhão de pessoas apresente excesso de peso, das quais, trezentos milhões são obesos. Projeções baseadas em inquéritos nacionais realizados nas últimas décadas estimam, que a obesidade atinja, em 2025, 40\% da população nos Estados Unidos, 30\%, na Inglaterra, e 20\%, no Brasil. (CONDE e BORGES, 2011).

Alguns autores obtiveram o mesmo achado. (ORNELA et al., 2016).

No artigo Acupuntura no tratamento da obesidade, observou redução no IMC de $67 \%$ e CA de $78 \%$ no grupo de tratamento 1, redução no IMC em $56 \%$ e de circunferência abdominal em $78 \%$ no grupo de tratamento 2 e a redução no IMC em $89 \%$ e de circunferência abdominal em $67 \%$ no grupo controle. (BONIZOL et al., 2016). No artigo tratamento da obesidade em auriculoterapia: relato de casos desenvolveram um estudo experimental, de caráter prospectivo e transversal, apresentado na forma de relato de casos, onde quatro indivíduos de ambos os sexos foram selecionados e datados como "A, B, C, e D", com base no índice de massa corporal (IMC>25). os autores empregaram a técnica de aurículocibernética, considerando a descrição específica para cada ponto, que consiste na inserção de agulhas inoxidáveis semipermanentes na orelha dominante. $O$ intervalo entre as sessões foi de uma semana, sendo que um ciclo de tratamento correspondeu a oito sessões completas. Como resultado, os indivíduos $A$ e $B$ foram os que mais perderam peso, em média $2,75 \mathrm{Kg}$, em seguida, a paciente $D$ com $1,6 \mathrm{Kg}$ e por último o paciente $C$ que perdeu 700 


\section{Universidade do Extremo Sul Catarinense \\ Revista Ibero-Americano de Humanidades, Ciências e \\ Educação \\ Unesc Produção e democratização do conhecimento na lbero-América}

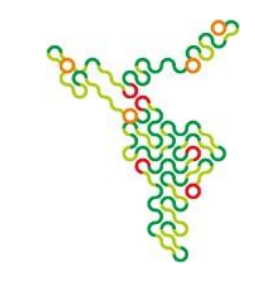

gramas. Concluíram que conjunto de pontos auriculares foi efetivo na redução do peso corporal.

De acordo com o estudo de (FRANZONI, COSTA e SANTOS, 2007), a auriculoterapia tem um efeito positivo na melhora de sintomas emocionais relacionados com a obesidade, como a ansiedade; melhoria do bem-estar geral, aumentando a disposição e dinamismo, fatores importantes para a perda e controle do peso, (SCHNEIDER e RODRIGUES, 2018), realizou estudo quantitativo com 15 participantes, sendo que destas $40 \%$ estavam sobrepeso, $20 \%$ estavam com obesidade grau I e $40 \%$ estavam com obesidade grau II, todos do sexo feminino, constatou-se neste estudo, que houve uma redução estatisticamente significativa na média do peso corporal, das medidas de circunferências da cintura, quadril e abdome. A diferença média de redução de peso foi $1,9 \mathrm{Kg}$, circunferência da cintura teve uma redução na média de $2,6 \mathrm{~cm}$, do quadril uma diminuição de $1,9 \mathrm{~cm}$, e do abdome de $2,5 \mathrm{~cm}$, também o apetite reduziu 0,9 pontos. Alguns estudos demonstram a eficácia da acupuntura e que incentivam a realização de novos estudos

\section{CONSIDERAÇÕES FINAIS}

É importante realçar que a obesidade caracteriza a complexidade dos problemas sociais, em especial na saúde pública com enfoque multiprofissional, e a auriculoterapia é uma Prática Integrativa e Complementar que vem sendo indicada nas Políticas Públicas de saúde para tratamento de obesidade e de outras condições cônicas, este trabalho evidenciou alterações na diminuição do apetite e redução do peso, mas ainda existe poucos estudos.

O profissional que trabalha com Atenção Básica é essencial que conheça as Práticas Integrativas e Complementares no SUS, e apliquem nos serviços como tratamento complementar, sendo essencial o apoio ao autocuidado com estímulo a mudança de hábitos como atividade física e alimentação saudável. 


\section{Universidade do Extremo Sul Catarinense}

Revista Ibero-Americano de Humanidades, Ciências e

Educação

UneSC Produção e democratização do conhecimento na lbero-América

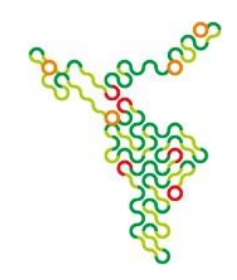

\section{APÊNDICE A}

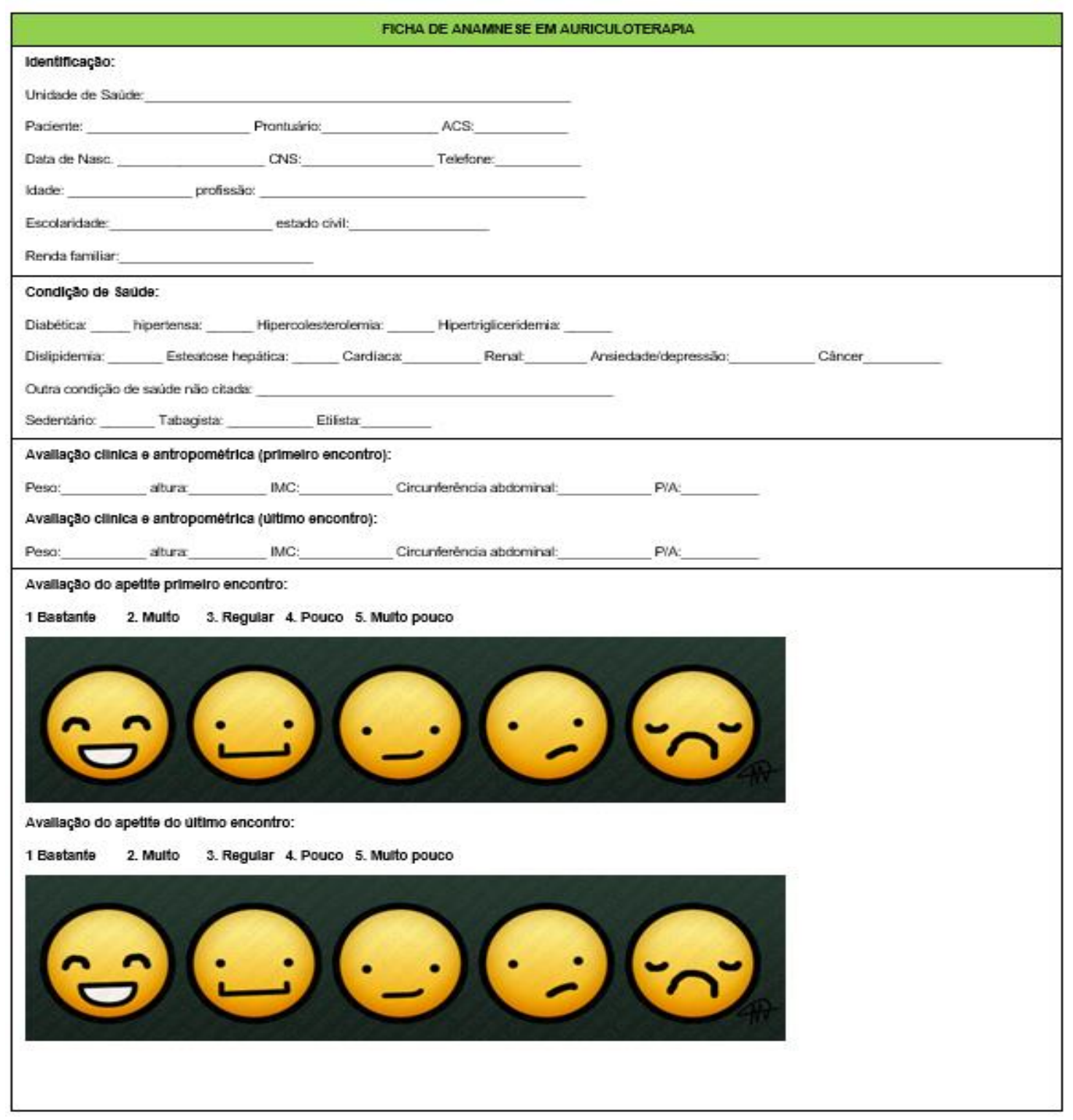

\section{REFERÊNCIAS BIBLIOGRÁFICAS}

ABESO- Associação Brasileira para o Estudo da Obesidade e da Síndrome Metabólica. Diretrizes Brasileira de Obesidade. AC Farmacêutica. Revista da ABESO, São Paulo. v.3, 2009. Disponível em: <http://www.abeso.org.br/pdf/diretrizes_brasileiras_obesidade_2009_. Acesso em 11 de ago. de 2019.

BONIZOL, W.; SALVI, O. J.; VALIATTI, B. T.; DULCIN, M. Tratamento da obesidade com auriculoterapia. Relato de caso. 10.18606/2318-1419/amazonia.sci.health. Revista Amazônia Science \& Health. V.4, n.3, p.19-24, 2016. Disponível em:<file:///C:/Users/Nagem/Downloads/1108-4931-1-PB.pdf.>. Acesso em 11 de

Revista Ibero-Americana de Humanidades, Ciências e Educação- Criciúma, v.5. n.3, 2019 


\section{Universidade do Extremo Sul Catarinense \\ Revista Ibero-Americano de Humanidades, Ciências e \\ Educação}

Unesc Produção e democratização do conhecimento na llbero-América

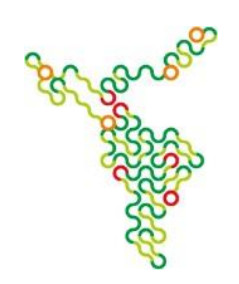

agosto de 2019.

BRASIL. Ministério da Saúde. Política Nacional de Alimentação e Nutrição. Revista ABED. Brasília- DF, 2.a edição, 2003. Disponível em:<http://www.abed.org.br/congresso2017/trabalhos/pdf/452.pdf>. Acesso em 11 de agosto de 2019.

BRASIL. Ministério da Saúde. Secretaria de Atenção à Saúde. Departamento de Atenção Básica. Política Nacional de Práticas Integrativas e Complementares no SUS - PNPIC-SUS. Brasília: Ministério da Saúde; 2006. Disponível em:<http://bvsms.saude.gov.br/bvs/saudelegis/gm/2006/prt0971_03_05_2006.html>.

Acesso em 16 de julho de 2019.

BRASIL. Ministério da Saúde. Práticas Integrativas e Complementares em Saúde: uma realidade no SUS. Revista Brasileira Saúde da Família. Ano IX, Ed. Especial (Maio 2008). Brasília: Ministério da Saúde, 2008. 76p.

BRASIL. Ministério da Saúde (MS). Secretaria de Atenção à Saúde. Departamento de Ações Programáticas Estratégicas. Política nacional de atenção integral à saúde do homem: princípios e diretrizes. Brasília, Editora do Ministério da Saúde, 2009. Disponível

em http://bvsms.saude.gov.br/bvs/publicacoes/politica_nacional_atencao_saude_home m.pdf>. Acesso em 11 de agosto de 2019.

BRASIL. Ministério da Saúde. Secretaria de Atenção à Saúde. Departamento de Atenção Básica. Práticas integrativas e complementares: plantas medicinais e fitoterapia na Atenção Básica/Ministério da Saúde. Secretaria de Atenção à Saúde. Departamento de Atenção Básica. - Brasília: Ministério da Saúde, 2012. 156 p.: il. Série A. Normas e Manuais Técnicos. Cadernos de Atenção Básica, n. 31. Disponível

em<http://bvsms.saude.gov.br/bvs/publicacoes/praticas_integrativas_complementares plantas_medicinais_cab31.pdf>.Acesso em 11 de agosto de 2019.

CONDE, W. L.; BORGES, C. O risco de incidência e persistência da obesidade entre adultos brasileiros segundo seu estado nutricional ao final da adolescência. Revista Bras. Epidemiol. (Supl.), p. 71-89, 2011.

DIAS, P.C.; HENRIQUES, P.; ANJOS, L. A.; BURLANDY, L. Obesidade e políticas públicas: concepções e estratégias adotadas pelo governo brasileiro. Cad. Saúde Pública 33(7), 2017. Disponível em<http://www.scielo.br/pdf/csp/v33n7/1678-4464csp-33-07-e00006016.pdf>. Acesso em 11 de agosto de 2019.

ERRAZ, D.; KRAICZYK, J. Gênero e políticas públicas de saúde - construindo respostas para o enfrentamento das desigualdades no âmbito do SUS. Revista de Psicologia da UNESP, 9(1), p. 70-82, 2010.

FRANZONI, L.; COSTA, T. V; SANTOS, M. C. Auriculoterapia em caso de obesidade grau I. Tecnologia em Cosmetologia e Estética, da Universidade do Vale do Itajaí. Balneário do Camboriú-SC, 2007.

GOMES, R.; MOREIRA, M. C. N; NASCIMENTO, E.F.; RABELLO, L. E. F. S; COUTO, M. T. SCHRAIBER, L. B. Os homens não vêm! Ausência e/ou invisibilidade masculina na atenção primária. Ciência \& Saúde Coletiva, 16. (Supl. 1), p. 983-992, 2011. Disponível em:< https://www.scielosp.org/pdf/csc/2011.v16suppl1/983-992/pt>. Acesso 


\section{Universidade do Extremo Sul Catarinense \\ Revista lbero-Americano de Humanidades, Ciências e \\ Educação \\ UneSC Produção e democratização do conhecimento na lbero-América}

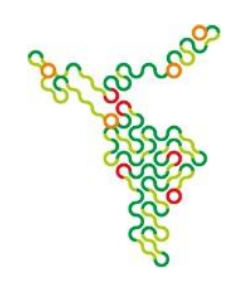

em 11 de agosto de 2019.

HANS-ULRICH, H. Atlas colorido de acupuntura: pontos sistêmicos, pontos auriculares e pontos gatilhos. Guanabara Koogan: Rio de Janeiro, 2007.

HONG, J. P. Métodos de Acupuntura e Manipulações. São Paulo: Roca, 2005.

JÚNIOR, E.T. Práticas integrativas e complementares em saúde, uma nova eficácia para o SUS. v. 30, n. 86, São Paulo, Jan./Apr., 2016. Disponível em:<http://www.scielo.br/scielo.php?script=sci_arttext\&pid=S01034014201600010009 9>. Acesso em 17 de agosto de 2019.

LEVIN, J. S; JONAS, W. B, editores. Tratado de medicina complementar e alternativa. São Paulo: Manole, 2001.

NEVES, M. L. Manual Prático de Auriculoterapia. Porto Alegre: Merithus. Organização Mundial de Saúde- OMS [homepage na internet]. Health topics: obesity, 2014. Disponível em:<http://books.scielo.org/id/rfdq6/pdf/anjos-9788575413449.pdf>. Acesso em 11 de agosto de 2019.

ORNELA, R. G.; OBA, M. V.; KNOUCHI, F. L.; SIGOLI, M. A.; SCANDIUZZI, R. J.; SOARES, D. W.; CARVALHO, P.C. Acupuntura no tratamento da obesidade. J Health Sci Inst.34(1), p.17-23, $2016 . \quad$ Disponível em:<https://www.unip.br/presencial/comunicacao/publicacoes/ics/edicoes/2016/01_janmar/V34_n1_2016_p17a23.pdf>. Acesso em 13 de agosto de 2019.

ROMOLI, M. Diagnóstico da Acupuntura Auricular. São Paulo: Roca, 2013.

SILVA, P. R.; BERTAN, H.; SENNA, V.S. Acupuntura Auricular. São Paulo: Phorte, 2012.

SILVA, J. P; PEREIRA, L; ASSIS, I, B. A auriculoterapia no tratamento de ansiedade e obesidade. Rev. Saúde em Foco. Ed. Minas Gerais, v. 10ํㅡ, 2018.

SOUSA, I. M. C.; VIEIRA, A. L. S. Serviços Públicos de saúde e medicina alternativa. Revista Ciência e Saúde Coletiva, v. 10, p. 255-266, 2005.

SOUSA, I. M. C; TESSER, C. D. Medicina Tradicional e Complementar no Brasil: inserção no Sistema Único de Saúde e integração com a atenção primária. Cad. Saúde Pública, V. 33, 1, 2017. Disponível em:< http://www.scielo.br/pdf/csp/v33n1/1678-4464-csp-33-01-e00150215.pdf>. Acesso em 11 de agosto de 2019.

SCHNEIDER, C.; RODRIGUES, D. M. Efeitos da auriculoterapia no tratamento do sobrepeso e obesidade. Estética e Bem-Estar. Tubarão, 2018. Disponível em:<https://www.riuni.unisul.br/bitstream/handle/12345/5497/TCCAURICULOTERAPIA \%282\%29.pdf?sequence=1\&isAllowed=y. Acesso em 11 de agosto de 2019. 